\title{
What feeds the green-eyed monster: sociodemographic and sociosexual determinants of jealousy
}

\author{
IVANA HROMATKO $^{1 *}$, MARTA FAJFARIĆ ${ }^{2}$ and MERI TADINAC ${ }^{1}$ \\ ${ }^{1}$ Department of Psychology, University of Zagreb, Faculty of Humanities and Social Sciences, I. Lucica 3, 10000 Zagreb, Croatia \\ ${ }^{2}$ First Gymnasium, Second Gymnasium, Varaždin, Croatia
}

(Received: 23 Apr 2019; accepted: 13 Oct 2019)

\begin{abstract}
Although studies consistently show gender differences in emotional vs. sexual jealousy, a substantial part of variance in jealousy is left unexplained. Here, we present two studies with aim to explore other correlates of jealousy, aside from gender. In the first online study $(n=2970)$, we found that participants who reported being more upset by the emotional infidelity scenario were older and more educated and had a higher income than those who reported being more upset by the sexual infidelity scenario. Those who expressed greater sexual jealousy gave higher ratings of importance of potential partner's mate value. Heterosexual women were more likely to report emotional jealousy than non-heterosexual women. Among men, sexual orientation did not predict type of jealousy. As the role of reproductive status was largely neglected in previous research, in the second study, we used a continuous measure to explore jealousy as a function of age (reproductive vs. post-reproductive; $n=199$ ). We found that the older participants were less jealous overall, and that the previously reported gender differences disappeared in the post-reproductive group. These results provide further support for the notion that jealousy is a context-specific, adaptive response, which diminishes in both intensity and specificity as the threat that it was designed for wanes.
\end{abstract}

Keywords: emotional vs. sexual jealousy, gender differences, reproductive status, context-specificity

\section{INTRODUCTION}

Oh, beware, my lord, of jealousy!

It is the green-eyed monster which doth mock.

The meat it feeds on.

-Shakespeare (Othello, 1622).

One of the main problems associated with achieving reproductive success is finding and retaining the right partner. Evolutionary psychologists postulate that jealousy is a psychological mechanism that helps us in the latter (Buss 2008). It can facilitate the recognition of subtle cues of partner's potential infidelity and activate the preventive behaviors. Approaches to jealousy are various: jealousy as a personality trait, jealousy as a specific emotion, jealousy as a set of actions, etc. In the context of the evolutionary problem of successful reproduction, jealousy can be best described as a complex psychological system that is activated when an individual perceives a threat, i.e. the possibility that another person might take his/her place in a romantic/sexual relationship. When this system is activated, it launches a series of actions aimed at removing the threat. Thus, the psychological mechanism of jealousy in the human evolutionary past solved the main threat to the individual's reproductive success - partner infidelity.

\section{Gender differences in reproductive strategies and jealousy}

It has been repeatedly shown that women exhibit a stronger preference than men do for attributes of ambition, social status, and financial wealth of their partner, which is indicative of the partner's ability to acquire and invest the resources necessary for the survival of the offspring, while men exhibit a stronger preference than women do for indicators of youthfulness and physical attractiveness, which are indicative of high reproductive potential, as well as for indicators of sexual fidelity (e.g. Buss 2008; Buss \& Schmitt 1993; Feingold 1992; Kenrick \& Keefe 1992; Kenrick et al. 1990; Wiederman \& Allgeier 1992). Both men and women rate some attributes, such as mutual attraction/love and health, as very important. However, to find a partner with desirable characteristics is not enough - he/she must be retained, at least for a certain period, to enable the individual to achieve reproductive success.

The main threat to this goal is the possibility of the partner's infidelity, which can take a form of either a short-term sexual infidelity (i.e., a one-night stand) or a long-term commitment to another person. As the possibility of losing a mate can be regarded as a serious adaptive problem, it is reasonable to expect that selection has favored the solutions, in the form of emotional and behavioral responses intended for mate retention. Social scientists exploring the psychology of jealousy have addressed the issues of the magnitude and/or frequency of jealousy and found no gender differences (e.g., DeSteno \& Salovey 1996). Those studies have been criticized

*Author for correspondence. E-mail: ihromatk@ffzg.hr.

This is an open-access article distributed under the terms of the Creative Commons Attribution-NonCommercial 4.0 International License (https://creativecommons.org/licenses/by-nc/4.0/), which permits unrestricted use, distribution, and reproduction in any medium for non-commercial purposes, provided the original author and source are credited, a link to the CC License is provided, and changes - if any - are indicated. 
(Buss 2008) for posing the question in too global a manner, ignoring the possible gender differences in cues that could trigger jealousy. If we consider jealousy as a psychological mechanism intended for solving the adaptive problems of infidelity in reproductive relationships (Daly, Wilson, \& Weghorst 1982; Wilson \& Daly, 1992), then it should have evolved differently for men and women because they were faced with different infidelity types, threatening their reproductive success. While the female is always certain that her offspring carries her genes, a male lacks paternity certainty. Female's infidelity can hence deprive him of a reproductive opportunity and burden him with high costs of cuckoldry as he invests in a genetically unrelated offspring (Buss 1999; Buss \& Schmitt 1993; Feingold 1992; Waynforth \& Dunbar 1995). We might therefore expect men to be more jealous about their partner's potential sexual infidelity. The female's adaptive problems concerning partner infidelity were somewhat different. A female faced no parenthood uncertainty, but if her partner turned his affections to another woman, she and her offspring stood to lose the resources and protection he provided. As the threat of losing a partner's commitment could be signaled by his emotional attachment to another female, we might expect women to be more concerned about their partner's emotional infidelity. This pattern of gender differences in jealousy was found in studies that utilize both verbal reports and physiological measures (Bohner \& Wänke 2004; Brase, Caprar, \& Voracek 2004; Buss et al. 1992; Buunk et al. 1996; de Souza et al. 2006; Frederick \& Fales 2016; Kruger et al. 2015; Pietrzak et al. 2002; Sagarin et al. 2012; Takahashi et al. 2006; Schützwohl 2004, 2005, 2007, 2008; Schützwohl \& Koch 2004; Wiederman \& Kendall 1999). Furthermore, it was shown that the actual infidelity experiences produced even more pronounced sex-typical differences when memories of these experiences were activated (Bendixen et al. 2015).

\section{The question of reproductive status: age and sexual orientation}

The majority of previous studies were conducted on young, heterosexual participants, usually from a convenience sample of (psychology) students. Only a limited number of studies involved other age groups, and their findings were inconsistent (Edlund et al. 2006; Green \& Sabini 2006; Lantagne \& Furman 2017; Shackelford et al. 2004). Recently, using the Articulated Thoughts in Simulated Situations paradigm, Babeva (2015) showed that older adults reacted less negatively in response to a scenario in which they unexpectedly witnessed their romantic partner interact in an intimate manner with an extradyadic individual. In an interesting study about sources of envy, Henninger and Harris (2015) showed that older participants were less likely to report being envy of someone's romantic success. Reaching post-reproductive age removes some of the selection pressures generating gender differences in jealousy among individuals of reproductive age, and one might postulate that people in older age groups do not show the same patterns of jealousy responses.
Studies of jealousy in homosexuals are even fewer, and they were either not primarily focused on jealousy (Bailey et al. 1994), did not include women (Bringle 1995; Hawkins 1990), or were carried out on very small samples (de Souza et al. 2006). Harris (2002) showed that participants of both genders, irrespective of their sexual orientation, focused more on a mate's emotional infidelity than on a mate's sexual infidelity. Bevan \& Lannutti (2002) showed that expression of jealousy varied by sexual orientation and sex; however, the experience of cognitive and emotional jealousy did not significantly differ by either sexual orientation or sex. Neither of these studies included responses from participants who reported having a bisexual orientation. As the degree of sexual attraction to either men or women varies, we think it is important to include bisexual participants as well, in order to explore their mating preferences and jealousy patterns and compare them with those of heterosexual and homosexual participants. Some authors have suggested that because those mechanisms had been designed by natural selection to promote reproductive fitness, none of these differences should be evident in non-reproducing groups of individuals (Sheets \& Wolfe 2001). Indeed, the responses of heterosexual participants vary depending on the type of infidelity (different-sex vs. same-sex infidelity) being involved (e.g., Denes, Lannutti, \& Bevan 2015; Mellgren et al. 2010).

Others consider sexual orientation as a powerful tool for testing hypotheses regarding sex differences and answering various questions about the nature of human mating dynamics (e.g., Rahman \& Wilson 2003; Rahman 2005; Bao \& Swaab 2011).

\section{The gender differences are not absolute}

Additionally, even though the majority of studies consistently showed gender differences in jealousy, those differences are never absolute: some men report being more upset by the scenario evoking emotional jealousy, and some women exhibit more jealousy over the possible sexual infidelity of their partners. The largest gender differences are usually reported in the US samples (e.g., 70\% men vs. $17 \%$ women being more upset by the idea of their partner's sexual infidelity; Buss et al. 1992), while the differences in European samples tend to be smaller, and sometimes both genders report being more upset by the emotional infidelity scenarios (Brase, Caprar, \& Voracek 2004; de Souza et al. 2006; Buunk et al. 1996; Mellgren et al. 2010; Schützwohl \& Koch 2004; Wiederman \& Kendall 1999; Zandbergen \& Brown 2015).

Some of these variations might originate from differences in appraisal processes (Mesquita \& Frijda 1992). Thus, different behaviors and types of infidelities might be appraised differently across cultures: for example, flirting might be considered a greater offense than kissing in some cultures, and the opposite can be found in others (Buunk \& Hupka 1987). A recent study, using a more complex measure of jealousy, showed that men and women perceived partner's involvement in a cross-sex friendship equally threatening the existence of the romantic relationship (Worley \& Samp 2014). Obviously, variables other than gender influence the variability of 
responses. Therefore, the aim of the two studies that we present here was to explore some correlates of jealousy that have previously been neglected - mostly the reproductive status of the participants, but also some sociodemographic characteristics, such as education and income, as well as some sociosexual characteristics, such as the pursuit of short- vs. long-term relationship, sexual orientation, and their mate preferences.

\section{STUDY 1}

Here we opted to explore some of the within-gender differences in jealousy. Specifically, we investigated the relationship between the emotional vs. sexual jealousy and some relevant aspects of mating:

1 Are there differences in demographic characteristics of individuals more prone to sexual or emotional jealousy?

2 Is there a difference in the type of relationship (long vs. short term) that sexually or emotionally jealous individuals are currently looking for?

3 Are there differences in the preferred mate's characteristics between sexually and emotionally jealous individuals?

4 Is the pattern of emotional vs. sexual jealousy responses the same among individuals of different sexual orientations?

\section{Method}

Procedure and questionnaires. To address these questions, we used an online three-part questionnaire consisting of three sections. Participants were solicited via banners posted on several popular internet portals in Croatia. The first section requested biographical data, including age, gender, income, marital status, sexual orientation, and preference for a long-term and a short-term relationship.

The second section was a list of characteristics of a potential mate (adapted from Buss et al. 1990). For the purposes of this study, we examined six characteristics: four that usually show the greatest gender differences regarding their desirability in a potential mate (favorable social status and good financial prospect favored by women and good looks and chastity favored by men), and two that are usually rated as very important by both genders (Mutual attraction-love and Health). Participants were asked to rate the importance and desirability of each characteristic on a 4-point scale (from 1 meaning "irrelevant" to 4 meaning "essential").

The third section included a hypothetical situation that could provoke either emotional or sexual jealousy, and the participants were asked to choose between one of those outcomes (adapted from Buss et al. 1992). The instruction was as follows: "Think of a relationship that you have had in past, have now, or would like to have. Imagine that you have just discovered that your partner is interested in someone else. Which would upset or hurt you more: a) the thought of your partner being emotionally involved with that person, or b) the thought of your partner being sexually involved with that person?"
Participants. A total of 3023 participants completed the questionnaire. After removing invalid entries (incomplete questionnaires, identical entries, inconsistencies in answers, etc.), and those who reported being younger than 16 years old, 2970 subjects remained. Among them, 2655 reported being heterosexual (1682 women and $973 \mathrm{men}$ ), 182 reported being bisexual (132 women and $50 \mathrm{men}$ ), and 133 of them reported being homosexual ( 77 men and 56 women). Respondents were from a wide range of educational, socio-economical, and age groups. Their age span was 16-62 years, mean age being $27.8(\mathrm{SD}=6.6)$ years. They had different educational backgrounds: $18.5 \%$ of our respondents had only high-school education, 30.1\% of them were undergraduate students, and $51.4 \%$ had a university degree or higher (Master/PhD).

\section{Results}

Are there differences in demographic characteristics of individuals more prone to sexual or emotional jealousy?. As expected, more women than men found the emotional infidelity scenario to be more upsetting $\left(\chi^{2}=179.33\right.$, $p<0.001)$. However, the majority of both genders $(70.7 \%$ of men and $90.8 \%$ of women) found the emotional infidelity scenario to be more upsetting.

Women who reported being more upset by the scenario of emotional infidelity were older $(F=4.61, p<0.03)$, more educated $(F=2.51, p<0.05)$, and had higher income $(F=7.91, p<0.01)$ compared to those who reported sexual infidelity as more upsetting. Men who reported being more upset by the scenario of emotional infidelity were also older $(F=5.11, p<0.03)$, and more educated $(F=9.53, p<0.01)$, but did not differ in income $(F=0.93$, n.s. $)$ from those who reported sexual infidelity as more upsetting.

Is there a difference in the type of relationship (longvs. short-term) that sexually or emotionally jealous individuals Are currently looking for?. In order to determine whether participants who reported being more upset with sexual vs. emotional infidelity differ in the type of relationship they are currently interested in, we calculated 2 chi-squares. In women, there was no relation between the type of romantic relationship they were interested in and the type of infidelity they found more upsetting $\left(x^{2}=2.38\right.$, n.s): $90 \%$ of those interested in a long-term relationship and $88 \%$ of those interested in a short-term relationship would be more upset by the emotional infidelity. The difference was significant in men: $80 \%$ of men interested in a long-term relationship reported they would be more upset by emotional infidelity, as compared to $69 \%$ of men interested in a short-term relationship $\left(\chi^{2}=12.41\right.$, $p<0.001)$.

Are there differences in the preferred mate's characteristics between sexually and emotionally jealous individuals?. In order to determine whether participants who reported being more upset with sexual vs. emotional infidelity differ in the assessments of importance of certain partner's features, we conducted a multivariate analysis of variance (MANOVA) with gender and the infidelity type as independent variables and potential partner's characteristics as dependent variables. MANOVA showed 
Table 1. Between-subjects effects for the ANOVAs with gender and infidelity type as the independent variables and potential partner's characteristics as the dependent variables

\begin{tabular}{|c|c|c|c|c|c|}
\hline Source of variance & Dependent variable & $d f$ & $F$ & $p$ & Partial eta squared \\
\hline \multirow[t]{6}{*}{ Gender } & Good financial prospects & \multirow{12}{*}{$2606 / 1$} & 247.57 & 0.001 & 0.087 \\
\hline & Chastity & & 34.28 & 0.001 & 0.013 \\
\hline & Favorable social status & & 96.95 & 0.001 & 0.036 \\
\hline & Good looks & & 99.62 & 0.001 & 0.037 \\
\hline & Health & & 1.42 & n.s. & 0.001 \\
\hline & Mutual attraction - love & & 39.40 & 0.001 & 0.015 \\
\hline \multirow[t]{6}{*}{ More upset by emotional vs. sexual infidelity } & Good financial prospects & & 15.07 & 0.001 & 0.006 \\
\hline & Chastity & & 27.53 & 0.001 & 0.01 \\
\hline & Favorable social status & & 3.70 & 0.05 & 0.001 \\
\hline & Good looks & & 14.99 & 0.001 & 0.006 \\
\hline & Health & & 3.07 & n.s. & 0.001 \\
\hline & Mutual attraction - love & & 2.55 & n.s. & 0.001 \\
\hline
\end{tabular}

significant main effects of gender $(F(2601,6)=96.49$, $\left.p<0.001, \eta p^{2}=0.182\right)$ and type of infidelity $\left(F(2601,6)=8.54, p<0.001, \eta p^{2}=0.019\right)$, without interaction $(F(2601,6)=0.60$, n.s. $)$. Between-subject effects are shown in Table 1.

Women valued good financial prospects, favorable social status, and mutual attraction-love more than men did, and men valued chastity and good looks more than women did. No differences were found in ratings of importance of health. Those who expressed sexual jealousy gave higher importance ratings for good financial prospects, favorable social status, chastity, and good looks than those who expressed emotional jealousy. Post-hoc $t$-tests were conducted in order to determine which characteristics of a potential partner were evaluated differently within each gender by those who expressed emotional jealousy and those who expressed sexual jealousy. In both men and women, those were good financial prospects $\left(t_{\mathrm{men}}=2.29\right.$, $\left.p<0.02 ; t_{\text {women }}=2.72, p<0.01\right)$, chastity $\left(t_{\text {men }}=4.61\right.$, $\left.p<0.001 ; t_{\text {women }}=3.03, p<0.01\right)$, and good looks $\left(t_{\text {men }}=3.39, p<0.001 ; t_{\text {women }}=2.54, p<0.001\right)$. These effects can be seen in Figure 1.

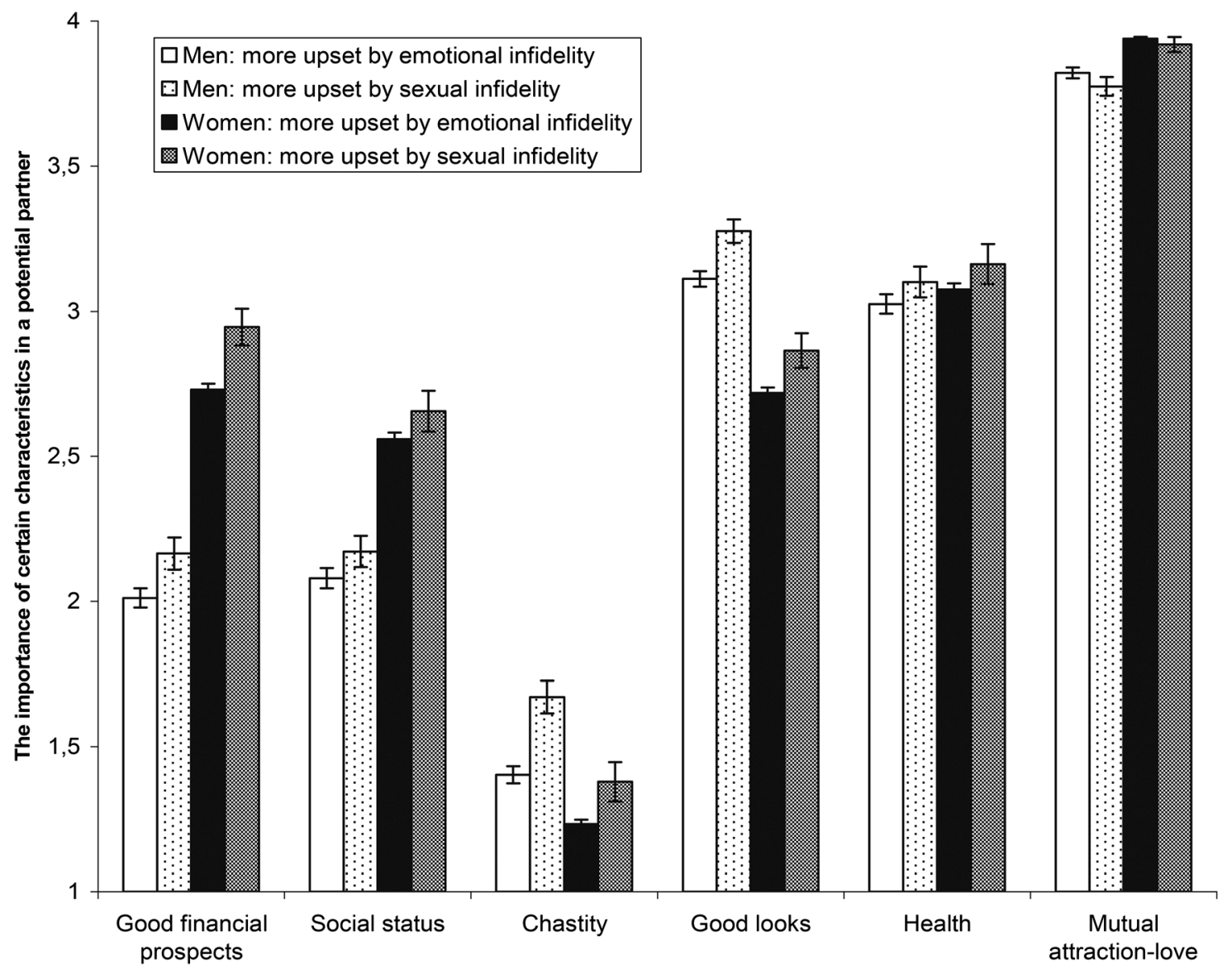

Figure 1. The importance of a potential partner's characteristics in heterosexual participants who reported being more upset by emotional vs. sexual infidelity 
Is the pattern of emotional vs. sexual jealousy responses the same among individuals of different sexual orientations?. Women of different sexual orientations showed very subtle differentiation when it comes to the type of infidelity that they find more upsetting, with heterosexual women having the highest rate of emotional jealousy responses $(90.8 \%)$, followed by bisexual $(86.8 \%)$ and homosexual (76.8\%) women $\left(\chi^{2}=13.78, p<0.001\right)$. Contrary to some previous reports, we found no statistically significant differences between males of different sexual orientations in the type of infidelity that they found more upsetting, although a certain trend can be noticed, with $80 \%$ of homosexual men reporting being more upset by emotional infidelity, comparing to $72 \%$ of bisexual and $70.7 \%$ of heterosexual men $\left(\chi^{2}=2.92, p=0.23\right)$.

\section{Discussion}

The results of our first study are consistent with the prediction that more heterosexual men $(29.3 \%)$ than heterosexual women $(9.2 \%)$ would express sexual jealousy, but if we look at the whole sample, irrespective of gender, it can be seen that the majority of participants reported being more upset by the idea of their partner's emotional infidelity $(83.5 \%)$. Our results are more similar to those obtained on German (Buunk et al. 1996; Schützwohl \& Koch 2004) and Romanian (Brase, Caprar, \& Voracek 2004) samples compared to the results obtained on US samples (Buss 1992; Buss et al. 1992). However, this pattern of results is not identical to the one we found earlier, in a sample of students, where $48.8 \%$ of men and $14.3 \%$ of women found sexual scenario to be more upsetting (Mellgren et al. 2010). Although it could be argued that cultural differences influence the strength of the adaptive response, the structure of the sample considering their demographic characteristics and current interest in long-term vs. short-term relationship should also be taken into consideration as possible explanations. Heterosexual women, who reported being more upset by the emotional infidelity scenario were older, had a higher education, and higher income than women who reported sexual infidelity as more upsetting. Heterosexual men who reported being more upset by the scenario of emotional infidelity were also older and more educated than men who reported sexual infidelity as more upsetting. Some authors (e.g., Wiederman, \& Allgeier 1993) have already pointed out that one of the problems in jealousy research is that the main body of evidence comes from studies on college students, and not on adults with an experience in long-term romantic relationships. Indeed, the stage of life or experience might play a role in reactions to infidelity. Harris (2000) found that for undergraduate women in her sample, the results depended on whether participants had been involved in a serious relationship. It can be assumed that college students are less likely to have been involved in such a relationship compared to older adults. Furthermore, as Green and Sabini (2006) pointed out, although the jealousy modules are innate, they might be sensitive to situational factors, such as the changes of the female's reproductive value. Buss et al. (1992) predicted the male sexual jealousy to diminish as the age of his mate increased, because of her lesser reproductive value.
Our second research problem aimed to extend this discussion with regard to the type of relationship (short term vs. long term) the respondents are currently interested in. The percentage of women who reported being interested in a long-term relationship was not related to the type of infidelity they reported as more upsetting; interest in a long-term relationship was extremely high in both-those who considered emotional infidelity, and those who considered sexual infidelity the worse alternative. In men however, the proportion of those who reported being interested in a long-term relationship was significantly higher among those who would be more upset by emotional infidelity compared to those who would be more upset by sexual infidelity. It seems that, at least in men, the type of jealousy being expressed might depend upon the relationship context. This finding is especially interesting if we take into consideration that our participants were not asked to think about a certain type of relationship when they had to choose the infidelity scenario that made them more jealous; the question about their current preference for the short- vs. long-term relationship was embedded in the first section of the questionnaire, among the standard demographic variables.

Our third research problem pertained to the mating preferences, i.e., the importance of certain characteristics of a potential partner depending on gender and the type of expressed jealousy. MANOVA showed significant main effects of both gender and type of jealousy expressed. As mentioned before, gender differences are not the central issue in this paper, as they are discussed in more detail elsewhere (e.g. Buss \& Schmitt 1993; Buss 2008; Feingold 1992; Gangestad \& Simpson 2000; Kenrick \& Keefe 1992; Kenrick et al. 1990; Wiederman \& Allgeier 1992), but they do show a typical pattern predicted by evolutionary psychology theorists (see Figure 1): women assessed good financial prospects and social status as more important than men did, while men assessed good looks and chastity as more important than women did. As expected, health is valued equally by both genders. Women rated mutual attraction and love somewhat higher than men did, but this item had generally very high ratings of importance in both genders. However, the finding that is more essential for the aim of this study is that, within both genders, the participants who expressed sexual jealousy gave higher ratings of importance for good financial prospects, chastity, and good looks than those who expressed emotional jealousy. Interestingly enough, there were no differences in ratings of importance of health and mutual attraction (two features that are considered neutral in terms of sexual bias) depending on the type of expressed jealousy. Human mating is characterized by strategic pluralism (Gangestad \& Simpson 2000). The finding that individuals who pursue partners with higher mate value are also more attuned to the threats of potential sexual infidelity might reflect previous relationship experiences with such partners, or a potential unconscious expectation.

Finally, we addressed the notion that gender-typical responses might not be found in non-heterosexual samples. Among women of different sexual orientations, homosexual women showed the most male-like patterns of jealousy, while results of bisexual women fall somewhere in 
between those of homosexual and heterosexual women. This would be in accordance with the neurodevelopmental theory, according to which, the etiogenesis of sexual orientation is closely connected to the sexual differentiation of the brain (Ellis \& Ames 1987). In view of this theory of sexual orientation, heterosexuals and homosexuals should differ in particular neural structures regulating sexual orientation. However, it is feasible to suppose that those neural differences also affect other aspects of both sexual and nonsexual behavior patterns. And indeed, it seems that homosexual women are exposed to a greater degree of masculinisation than heterosexual women, but homosexual men turn out to be either over- or under-masculinised, depending on the trait being studied (for a review, see Rahman \& Wilson 2003). Men of different sexual orientations in our sample did not differ significantly regarding jealous reactions. We observed a non-significant trend toward somewhat higher incidence of emotional jealousy among homosexual men $(80 \%)$. However, both genders, across all sexual orientations, more often found the notion of emotional infidelity more upsetting than the notion of sexual infidelity. If we look at gender differences within a particular sexual orientation, they remained significant in heterosexual and bisexual populations, but disappeared in the homosexual sample, where $80.0 \%$ of men and $76.8 \%$ of women stated that they found emotional infidelity scenario more upsetting, similarly to the findings of Sheets and Wolfe (2001).

Previous research found that both homosexual men and women show some sex-atypical patterns of functioning, especially when it comes to childhood sex-typed behaviors and interests, but tapping into their mating preferences yielded inconsistent results (Bell, Weinberg, \& Hammersmith 1978; Green 1987; De Souza et al. 2006). Sheets and Wolfe (2001) suggested that sex differences in jealousy should disappear in non-reproducing groups, as those differences had been designed by natural selection to promote reproductive fitness. However, it is not feasible to presume homosexuality to undermine other sexual adaptations, as homosexuality is not adaptive in itself, but seems to involve biological mechanisms that are carried by sexual reproduction (Kenrick et al. 1995).

Finally, as with other studies using online methodology, there might be various methodological constraints, such as the bias in sampling process. Although such a bias surely exists, the participants in this study reported to be from various socio-economic backgrounds, having different levels of education, and their age span is wide. Thus, although the generalizability is limited, the findings are probably more generalizable than the typical findings from college students. As evolutionary psychology postulates that some cognitive mechanisms should be universal (universality of gender differences is, in fact, one of the strongest arguments against social deterministic explanations of these differences), the potential biases in sampling process should not have affected these results.

\section{STUDY 2}

The aim of this study was to further explore the role of age (reproductive vs. post-reproductive) and gender in different types of jealousy (emotional vs. sexual). If we consider jealousy to be a psychological adaptation reducing the likelihood of infidelity in reproductive relationships, then it could be expected to lose its intensity in post-reproductive age.

\section{Method}

Participants. A total of 218 participants took part in the study. They were recruited using a snowball paradigm, with the help of student volunteers. Results from 19 of them were excluded from further analyses, because they gave incomplete answers or did not understand the task. Thus, the following results are based on responses from 100 women and 99 men: 104 of them were of reproductive age (52 women and 52 men; mean age $=22.92$, $S D=2.06$, age range: $18-28)$, and 95 of them were of post-reproductive age (48 men and 47 women; mean age $=51.12, S D=1.97$, age range: $43-67)$. The two age categories did not differ regarding the gender composition $\left(\chi^{2}=0.006 ; d f=1 ; N=199 ; p>0.05\right)$, and there were no age differences between men and women within a given age category (reproductive age group: $t=0.356$; $d f=102$; $N=104 ; p>0.05$; post-reproductive age group: $t=1.576$; $d f=93 ; N=95 ; p>0.05)$.

At the time of testing, $52.9 \%$ of younger and $85.3 \%$ of the older participants reported currently being in a longterm relationship or marriage. The younger sample consisted mostly of students, but not students of psychology $(82.7 \%)$, followed by high-school graduates $(9.6 \%)$ and university degree holders $(5.8 \%)$. Among older participants, $67.4 \%$ had a university degree, $31.6 \%$ finished high school and vocational schools, and $1.1 \%$ finished elementary school only.

$86.3 \%$ of the Older Participants and Only 1\% of the Younger Participants Reported Having Children (the Difference Being Significant $\chi^{2}=5.472 ; d f=1 ; N=199$; $p<0.05)$.

Procedure and questionnaire. The participants filled out the questionnaire on emotional and sexual jealousy (Voracek 2000, personal communication). The first part of the questionnaire consisted of questions related to general demographics (age, gender, education, employment, marital status, children, etc.). In the second part, participants were asked to imagine a committed romantic relationship that they have had in the past, currently have or would like to have. The participants then had to imagine that the person from that relationship was interested in someone else. They were then asked to assess on a continuous measure how jealous they would feel in response to different forms of either emotional or sexual infidelity on part of their partner ( 0 meaning the complete absence of jealousy and 6 maximal jealousy). Cronbach $\alpha$ coefficients were as follows: 0.955 for male participants, 0.947 for female participants, 0.919 for younger participants; 0.968 for older participants.

\section{Results}

Two scores were computed: emotional jealousy score (average of responses to all items relating to emotional 
Table 2. Results of $2 \times 2 \times 2$ MANOVA with gender and age as between-subjects sources of variance and type of infidelity as a withinsubject source of variance

\begin{tabular}{|c|c|c|c|c|}
\hline & $d f$ & $F$ & $p$ & Partial eta squared \\
\hline Type of infidelity & \multirow{6}{*}{$1 / 195$} & 1.80 & 0.182 & 0.009 \\
\hline Gender & & 0.343 & 0.559 & 0.002 \\
\hline Age (reproductive vs. post-reproductive) & & 4.25 & 0.041 & 0.021 \\
\hline Type of infidelity $\times$ gender & & 51.51 & 0.001 & 0.209 \\
\hline Type of infidelity $\times$ age & & 3.98 & 0.047 & 0.020 \\
\hline Type of infidelity $\times$ gender $\times$ age & & 18.36 & 0.001 & 0.086 \\
\hline
\end{tabular}

infidelity) and sexual jealousy score (average of responses to all items relating to sexual infidelity). The hypothetical range of scores varied between 1 (no jealousy) to 7 (maximal intensity of jealousy). A mixed model MANOVA with gender and age (reproductive vs. post-reproductive) as the between-subjects sources of variance and type of infidelity (emotional vs. sexual) as the within-subject source of variance showed a significant effect of age (with participants of post-reproductive age being less jealous overall), as well as several significant interactions: type of infidelity $x$ gender, type of infidelity $x$ age, and type of infidelity $x$ gender $\times$ age. Results are shown in Table 2 , and the nature of these interactions can be seen from Figure 2 .

Overall, women reported more emotional jealousy than men $(t(197)=3.177 ; p<0.01)$, and men reported more sexual jealousy than women did $(t(197)=1.977$; $p<0.05)$. Participants of post-reproductive age did not differ from younger participants in emotional jealousy $(t(197)=1.23 ; p>0.05)$, but they had lower scores on sexual jealousy $(t(197)=2.5 ; p<0.05)$. The triple interaction, however, shows that pattern of gender differences in emotional vs. sexual jealousy is not the same in different age groups. Women and men of post-reproductive age did not differ in either emotional $(t(93)=1.6 ; p>0.05)$ or sexual jealousy $(t(93)=0.265 ; p>0.05)$. This previously well-documented pattern was found only in participants of reproductive age, with women scoring higher on emotional jealousy than men $(t(102)=3.012 ; p<0.01)$ and men scoring higher on sexual jealousy than women $(t(102)=3.391 ; p<0.01)$

The within-gender post-hoc tests showed that younger men did not differ from older men in emotional jealousy; however, they scored significantly higher on sexual jealousy $(t(97)=3.371 ; p<0.01)$. Women from different age groups did not differ in either emotional or sexual jealousy scores.

\section{Discussion}

Age has been largely neglected in majority of the previous studies on gender differences in jealousy. Here, we showed that age is in fact an important variable that has both significant main effects and interactions with other variables of interest, such as type of jealousy and gender. Overall, younger participants showed higher levels of jealousy than the older ones. Given the theoretical function of jealousy

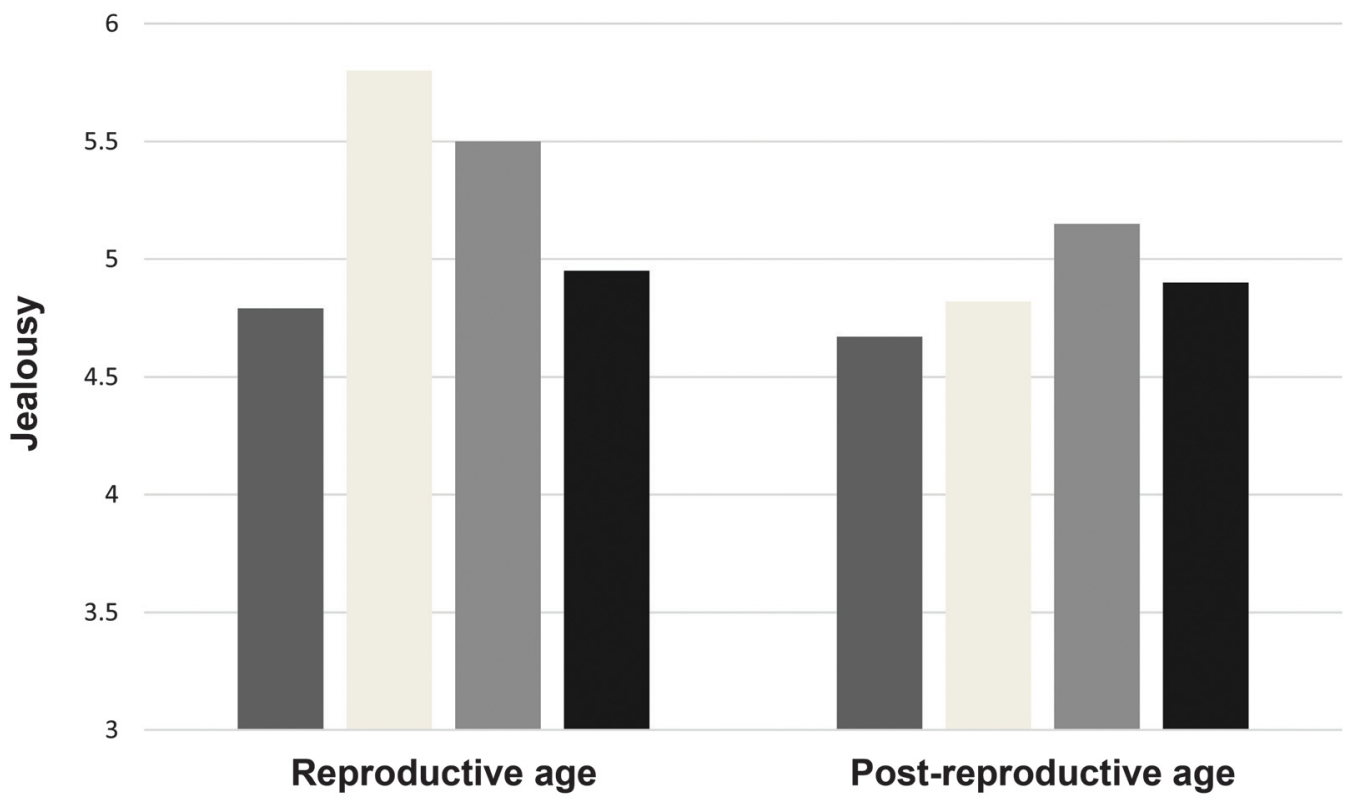

- Men: emotional infidelity

- Women: emotional infidelity
Men: sexual infidelity

-Women: sexual infidelity

Figure 2. Jealousy as a function of sexual vs. emotional infidelity in different age groups 
as psychological mechanism of infidelity prevention in reproductive relationships, it is possible that among older participants this functionality is lost: the majority of them $(68.3 \%$ in our study) already have children and even grandchildren. In addition, being of post-reproductive age, their partners' infidelity does not pose a huge threat to their reproductive success.

On the other hand, only $1 \%$ of younger participants already had children. Thus, infidelity in this age group represents a considerably more serious threat to individual's reproductive success. At the same time, individuals of reproductive age also have more to gain with infidelity (e.g. benefits of obtaining a partner with higher mate value). Both fertility and reproductive value decrease with woman's age (Fisher 1958). This can explain why older men show less sexual jealousy than younger men (assuming that their partners are of similar age as themselves). As men enter the post-reproductive period much later than women, their infidelity even at older age might result in new offspring and re-allocation of their resources. Still, this should not have a significant impact on his primary partner's reproductive success, as their children are likely grown enough to survive on their own, i.e., they do not need huge parental provision. Shackelford et al. (2004) found that older women reacted less negatively to partner's emotional infidelity as compared to younger women. Although older women in our study did show somewhat lower levels of emotional jealousy than younger ones, this difference did not reach statistical significance. Finally, personal experience with decreasing sexual power might result in reduced tendency of older participants to respond in a jealous manner - they might not believe that the infidelity scenario is likely to occur.

The most interesting finding of this study is the triple interaction between age, gender, and type of infidelity. The fact that the previously well-established pattern of gender differences (men being more sexually jealous and women being more emotionally jealous) appeared only among participants of reproductive age is in line with the notion that jealousy is an adaptive mechanism, but only in reproductive relationships. It diminishes in both intensity and specificity as the threat that it was designed for wanes.

Some researchers have argued that gender differences in jealousy are an artifact of forced choice methods (see DeSteno et al. 2002). This study, with continuous measures of emotional and sexual jealousy, adds to the growing body of literature confirming gender differences using alternative measures (Pietrzak et al. 2002, Sagarin et al. 2012; Bohner \& Wänke 2004; Brase, Caprar, \& Voracek 2004; Sagarin 2005; Takahashi et al. 2006; de Souza et al. 2006; Edlund et al. 2006; Buss 2008). In addition, we used a community sample, and were careful to avoid students of psychology, which we believe contributes to the generalizability of our findings. Major shortcoming of this study is the over-representation of highly educated participants in both samples. As we have shown in Study 1, education plays a role in jealousy, and thus it would be interesting to explore whether this pattern of results would appear in a socio-economically more diverse group of participants.

\section{GENERAL DISCUSSION}

A growing body of evidence shows that men and women perceive threats to their relationship differently, and that they respond to potential partner's infidelity differently. These differences have been confirmed in various cultures (Brase, Caprar, \& Voracek 2004; Buss 1999; de Souza et al. 2006; Wiederman \& Kendall 1999), which is considered a gold standard for testing evolutionary hypotheses. They have also been confirmed using various methods, such as forced choice vs. continuous measures (Bohner \& Wänke 2004; Brase, Caprar, \& Voracek 2004; de Souza et al. 2006; Pietrzak et al. 2002; Sagarin 2005), identification of negative emotions accompanying infidelity scenarios (Buss et al. 1992, Pietrzak et al. 2002; Schützwohl 2008; Mellgren et al. 2010), recall of infidelity-related cues (Schützwohl \& Koch 2004), likelihood of relationship resolution or forgiveness following infidelity (Shackelford, Buss, \& Bennett 2002), and physiological measures, such as facial muscle contractions and galvanic skin response (Buss et al. 1992; Harris 2000), as well as fMRI (Takahashi et al. 2006).

However, even though the direction of gender differences is stable (men get more upset than women do by sexual infidelity and women get more upset than men do by emotional infidelity), the size of these differences varied significantly across studies. Some men are more upset by the possibility of their partner's emotional infidelity, and some women are more upset about the possible sexual infidelity of their partners. Our studies have shown that the likelihood (study 1) or intensity (study 2) of emotional vs. sexual infidelity is associated with several demographic variables (such as age, education, and income), as well as with participant's mate preferences, sexual orientation, and the context of seeking a short-term vs. long-term relationship. To summarize, our first study showed that those who reported being more upset by the notion of emotional than sexual infidelity were somewhat older and had higher education and higher income. They assessed potential mate's characteristics, such as financial prospects, favorable social status, chastity, and good looks, as less important than those who reported being more upset by the notion of sexual infidelity. Men who reported being more upset by emotional than sexual infidelity were more often interested in a long-term relationship. Furthermore, gender differences in emotional vs. sexual jealousy were found in heterosexual and bisexual sample, but disappeared in homosexual sample, in line with the notion that gender-specific jealousy as an adaptive response does not exist where it has no function, i.e., in non-reproducing relationships. The idea that intensity of jealousy in general, as well as the pattern of gender differences in jealousy specifically, diminishes in non-reproducing context (i.e., in post-reproductive age) was further corroborated in our second study, where a previously widely reported gender difference in sexual vs. emotional jealousy appeared only among younger participants.

Taken together, these results suggest that romantic jealousy is an adaptive, context-specific response, determined by a constellation of factors other than gender, such as age, social status, current relationship, and mate 
preferences. Thus, explanations of patterns of gender differences (or lack thereof) in jealousy may gain from more in-depth analyses of relationship between gender and reactivity to various jealousy-inducing scenarios.

\section{REFERENCES}

Babeva, K. N. (2015). Age differences in jealousy: An ATSS examination of the role of attributions in the emotional reactions of older and younger adults in relationshipthreatening situations, University of Southern California, Unpublished doctoral dissertation.

Bailey, J. M.; Gaulin, S.; Agyei, Y., \& Gladue, B. A. (1994). Effects of gender and sexual orientation on evolutionarily relevant aspects of human mating psychology. J. Pers. Soc. Psychol., 66, 1081-1093.

Bao, A. M., \& Swaab, D. F. (2011). Sexual differentiation of the human brain: Relation to gender identity, sexual orientation and neuropsychiatric disorders. Front. Neuroendocrinol., 32, 214-226.

Bell, A. P.; Weinberg, M. S., \& Hammersmith, S. K. (1978). Homosexualities: A study of diversity among men and women. New York: Simon \& Schuster.

Bendixen, M.; Ottesen Kennair, L. E., \& Buss, D. M. (2015). Jealousy: Evidence of strong sex differences using both forced choice and continuous measure paradigms. Pers. Individ. Differ., 86, 212-216.

Bevan, J. L., \& Lannutti, P. J. (2002). The experience and expression of romantic jealousy in same-sex and oppositesex romantic relationships. Commun. Res. Rep., 19, 258268.

Bohner, G., \& Wänke, M. (2004). Priming of AIDS and reactions to infidelity: Are sex differences in jealousy context-dependent? Zeitschrift für Sozialpsychologie, 35, 107-114.

Brase, G. L.; Caprar, D. V., \& Voracek, M. (2004). Sex differences in responses to relationship threats in England and Romania. J. Soc. Person. Relationships, 21, 763-778.

Bringle, R. G. (1995). Sexual jealousy in the relationships of homosexual and heterosexual men: 1980 and 1992. Pers. Relat., 2, 313-325.

Buss, D. M.; Larsen, R. J.; Westen, D., \& Semmelroth, J. (1992). Sex differences in jealousy: Evolution, physiology and psychology. Psychol. Sci., 3, 251-255.

Buss, D. M. (1992). Mate preference mechanisms: consequences for partner choice and intrasexual competition. In: Barkow, J.; Cosmides, L.; Tooby, J. (Eds.). Adapted Mind (pp. 249266). New York: Oxford University Press.

Buss, D. M. (1999). Evolutionary psychology. Needham Heights, MA: Allyn \& Bacon.

Buss, D. M. (2008). Evolutionary psychology: The new science of the mind. Boston: Pearson.

Buss, D. M., \& Schmitt, D. P. (1993). Sexual strategies theory: An evolutionary perspective on human mating. Psychol. Rev., 100, 204-232.

Buss, D. M.; Abbot, M.; Angleitner, A.; Asherian, A.; Biaggio, A.; Blanco-Villasenor, A., et al. (1990). International preferences in selecting mates: A study of 37 cultures. $J$. Cross-Cultural Psychol., 21, 5-47.
Buunk, B., \& Hupka, R. B. (1987). Cross-cultural differences in the elicitation of sexual jealousy. J. Sex Res., 23, 12-22.

Buunk, B. P.; Angleitner, A.; Oubaid, V., \& Buss, D. M. (1996). Sex differences in jealousy in evolutionary and cultural perspective. Test from the Netherlands, Germany, and the United States. Psychol. Sci., 7, 359-363.

Daly, M.; Wilson, M., \& Weghorst, S. J. (1982). Male sexual jealousy. Ethol. Sociobiol., 2, 11-27.

Denes, A.; Lannutti, P. J., \& Bevan, J. L. (2015). Same-sex infidelity in heterosexual romantic relationships: Investigating jealousy-related emotions, relational outcomes, and communicative responses. Pers. Relat., 22, 414-430.

De Souza, A. A.; Verderane, M. P.; Taira, J. T., \& Otta, E. (2006). Emotional and sexual jealousy as a function of sex and sexual orientation in a Brazilian sample. Psychol. Rep., 98, 529-535.

DeSteno, D. A.; Bartlett, M. Y.; Braverman, J., \& Salovey, P. (2002). Sex differences in jealousy: Evolutionary mechanism or artifact of measurement? J. Pers. Soc. Psychol., 83, 11031116.

DeSteno, D. A., \& Salovey, P. (1996). Jealousy and the characteristics of one's rival: a self- evaluation maintenance perspective. Pers. Soc. Psychol. Bull., 22, 920-932.

Edlund, J. E.; Heider, J. D.; Scherer, C. R.; Farc, M.; Sagarin, B. J. (2006). Sex differences in jealousy in response to actual infidelity. Evol. Psychol., 4, 462-470.

Ellis, L., \& Ames, M. A. (1987). Neurohormonal functioning and sexual orientation: A theory of homosexualityheterosexuality. Psychol. Bull., 101, 233-258.

Feingold, A. (1992). Gender differences in mate selection preferences: A test ofthe parental investment model. Psychol. Bull., 112, 125-139.

Fisher, R. A. (1958). The genetical theory of natural selection. 2nd ed., Dover: New York.

Frederick, D. A., \& Fales, M. R. (2016). Upset over sexual versus emotional infidelity among gay, lesbian, bisexual, and heterosexual adults. Arch. Sex. Behav., 45, 175-191.

Gangestad, S. W., \& Simpson, J. A. (2000). The evolution of human mating: Trade-offs and strategic pluralism. Behav. Brain Sci., 23, 573-644.

Green, M. C.; Sabini, J. (2006). Gender, socioeconomic status, age, and jealousy: Emotional responses to infidelity in a national sample. Emotion, 6, 330-334.

Green, R. (1987). The "sissy boy syndrome" and the development of homosexuality. New Haven, CT: Yale University Press.

Henniger, N. E.; Harris, C. R. (2015). Envy across adulthood: The what and the who. Basic Appl. Soc. Psychol., 37, 303318.

Harris, C. R. (2000). Psychophysiological responses to imagined infidelity: The specific innate modular view of jealousy reconsidered. J. Pers. Soc. Psychol., 78, 1082-1091.

Harris, C. R. (2002). Sexual and romantic jealousy in heterosexual and homosexual adults. Psychol. Sci., 13, 7-12.

Hawkins, R. O. (1990). The relationship between culture, personality, and sexual jealousy in men in heterosexual and homosexual relationships. J. Homosexuality, 19, 67-84.

Kenrick, D. T., \& Keefe, R. C. (1992). Age preferences in mates reflect sex differences in reproductive strategies. Behav. Brain Sci., 15, 75-133.

Kenrick, D. T.; Keefe, R. C.; Bryan, A.; Barr, A, \& Brown, S. (1995). Age preferences and mate choice among 
homosexuals and heterosexuals: A case for modular psychological mechanisms. J. Pers. Soc. Psychol., 69, 11661172.

Kenrick, D. T.; Sadalla, E. K.; Groth, G., \& Trost, M. R. (1990). Evolution, traits, and stages of human courtship: Qualifying the parental investment model. J. Pers., 58, 97-116.

Kruger, D.; Fisher, M.; Fitzgerald, C.; Garcia, J.; Geher, G., \& Guitar, A. E. G. (2015). Sexual and emotional aspects are distinct components of infidelity and unique predictors of anticipated distress. Evol. Psychol. Sci., 1, 44-51.

Lantagne, A., \& Furman, W. (2017). Romantic relationship development: The interplay between age and relationship length. Developmental Psychology, 53, 1738-1749.

Mellgren, R. L.; Hromatko, I.; McArthur, D., \& Mann, M. A. (2010). A test of the evolutionary explanation of jealousy in the United States and Croatia. Soc. Res., 19, 915-931.

Mesquita, B., \& Frijda, N. H. (1992). Cultural variations in emotions: A review. Psychol. Bull., 112, 179-204.

Pietrzak, R. H.; Laird, J. D.; Stevens, D. A., \& Thompson, N. S. (2002). Sex differences in human jealousy: A coordinated study of forced-choice, continuous rating-scale, and physiological responses on the same subjects. Evol. Hum. Behav., 23, 83-94.

Rahman, Q. (2005). The neurodevelopment of human sexual orientation. Neurosci. Biobehav. Rev., 29, 1057-1066.

Rahman, Q., \& Wilson, G. D. (2003). Born gay? The psychobiology of human sexual orientation. Pers. Individ. Differ., 34, 1337-1382.

Sagarin, B. L. (2005). Reconsidering evolved sex differences in jealousy: Comment on Harris (2003). Pers. Soc. Psychol. Rev. 9, 62-75.

Sagarin, B. J.; Martin, A. L.; Coutinho, S. A.; Edlund, J. E.; Patel, L.; Skowronski, J. J., \& Zengel, B. (2012). Sex differences in jealousy: A meta-analytic examination. Evol. Hum. Behav., 33, 595-614.

Schützwohl, A. (2004). Which infidelity type makes you more jealous? Decision strategies in a forced-choice between sexual and emotional infidelity. Evol. Psychol., 2, 121-128.

Schützwohl, A. (2005). Sex differences in jealousy: the processing of cues to infidelity. Evol. Hum. Behav., 26, 288-299.

Schützwohl, A. (2007). Decision strategies in continous ratings of jealousy feelings elicited by sexual and emotional infidelity. Evol. Psychol., 5, 815-828.
Schützwohl, A. (2008). Relief over the disconfirmation of the prospect of sexual and emotional infidelity. Pers. Individ. Differ., 44, 668-678.

Schützwohl, A., \& Koch, S. (2004). Sex differences in jealousy: The recall of cues to sexual and emotional infidelity in personally more and less threatening context conditions. Evol. Hum. Behav., 25, 249-257.

Shackelford, T. K.; Buss, D. M., \& Bennett, K. (2002). Forgiveness or breakup: Sex differences in response to a partner's infidelity. Cognit. Emotion, 16, 299-307.

Shackelford, T. K.; Voracek, M.; Schmitt, D. P.; Buss, D. M.; Weekes-Shackelford, V. A.; Michalski, R. L. (2004). Romantic jealousy in early adulthood and in later life. Hum. Nat., 15, 283-300.

Sheets, V. L., \& Wolfe, M. D. (2001). Sexual jealousy in heterosexuals, lesbians and gays. Sex Roles, 44, 255-276.

Takahashi, H.; Matsuura, M.; Yahata, N.; Koeda, M.; Suhara, T., \& Okubo, Y. (2006). Men and women show distinct brain activations during imagery of sexual and emotional infidelity. NeuroImage, 32, 1299-1307.

Waynforth, D., \& Dunbar, R. I. M. (1995). Conditional mate choice strategies in humans - evidence from lonely hearts advertisements. Behaviour, 132, 125-139.

Wiederman, M. W., \& Allgeier, E. R. (1992). Gender differences in mate selection criteria: Sociobiological of socioeconomic explanation? Ethol. Sociobiol., 13, 115-124.

Wiederman, M. W., \& Allgeier, E. R. (1993). Gender differences in sexual jealousy: Adaptionist or social learning explanation? Ethol. Sociobiol., 14, 115-140.

Wiederman, M. W., \& Kendall, E. (1999). Evolution, sex, and jealousy: Investigation with a sample from Sweden. Evol. Hum. Behav., 20, 121-128.

Wilson, M., \& Daly, M. (1992). The Man Who Mistook His Wife for a Chattel. Barkow, U. J. H.; Cosmides, L. and Tooby, J. (Eds), The adapted mind: Evolutionary psychology and the generation of culture (pp. 289-322). New York: Oxford University Press Inc.

Worley, T. R.; Samp, J. (2014). Friendship characteristics, threat appraisals, and varieties of jealousy about romantic partners' friendships. Interpersona, 8, 231-244.

Zandbergen, D. L., \& Brown, S. G. (2015). Culture and gender differences in romantic jealousy. Pers. Individ. Differ, 72, $122-127$. 